\title{
Rotational dynamics of methyl groups in m-xylene
}

\author{
O. Kirstein ${ }^{\text {a) }}$ \\ Bragg Institute, Australian Nuclear Science \& Technology Organisation, Menai NSW 2234, Australia \\ and Institut für Festköperforschung, Forschungszentrum Jülich, D-52425 Jülich, Germany \\ M. Prager \\ Institut für Festköperforschung, Forschungszentrum Jülich, D-52425 Jülich, Germany
}

R. M. Dimeo

NIST Center for Neutron Research, Gaithersburg, Maryland 20889

\author{
A. Desmedt \\ Berlin Neutron Scattering Center, Hahn-Meitner Institut, D-14109 Berlin \\ and University Bordeaux I, 33405 Talence Cedex, France
}

(Received 23 October 2003; accepted 13 October 2004; published online 13 December 2004)

\begin{abstract}
Methyl group dynamics of $\mathrm{m}$-xylene was investigated by using incoherent inelastic and quasi-elastic neutron scattering. Inelastic measurements were carried out at the high flux backscattering spectrometer HFBS at the National Institute of Standards, quasi-elastic measurements at the time-of-flight spectrometer NEAT at the Hahn-Meitner-Institute. Rotational potentials are derived which describe the tunnel splittings, first librational, and activation energies of the two inequivalent $\mathrm{CH}_{3}$ groups. Indications for coupling of the methyl rotation to low-energy phonons have been found. The finite width of one tunneling transition at He temperature is described by direct methylmethyl coupling. The combined results of the experiments and the calculations allow a unique assignment of rotor excitations to crystallographic sites. (C) 2005 American Institute of Physics.
\end{abstract}

[DOI: $10.1063 / 1.1829037]$

\section{INTRODUCTION}

The fundamental quantities that determine the spatial arrangement of the atoms of a molecule and the dynamics are the intermolecular interaction potentials. An exact knowledge of these potentials allow one to calculate the crystal structure and lattice dynamics of any molecular system. Calculating the lattice dynamics, even if the crystal structure of a molecular system is known, is very complex and time consuming. However, the increasing power of computers combined with force-field and quantum chemistry moleculardynamics software allow the numerical investigation of the low-energy dynamics based on individual atom-atom interactions. Using this technique the methyl group dynamics in, e.g., paracetamol and acetanilide was modeled by Johnson et al. ${ }^{1}$ and in 2,6 dimethylpyrazine by Nicolai et al. ${ }^{2}$ Another possibility of addressing this problem is to model the dynamics by using simplified, semiempirical interaction potentials commonly known as transferable pair potentials (TPP). The most important point of this approach lies in its transferability which, of course, simplifies the theoretical description molecular crystals see, e.g., Ref. 3 for the dynamics of $\mathrm{CH}_{3}$ groups in 2-butyne or Ref. 4 for the dynamics in acetamide. In the present work we discuss the experimental results of

\footnotetext{
a) Author to whom correspondence should be addressed. Also at: Bragg Institute, Australian Nuclear Science \& Technology Organization, New Illawara Road, Lucas Heights, PMB1 NSW 2234, Australia. Telephone: +612-9717 9452. Fax: +61-2-9717 3606. Electronic mail: oki@ansto.gov.au
}

neutron scattering experiments, which provide detailed information on the potentials determining the dynamics of the methyl groups.

Before beginning to describe the experimental results we recall some properties of $\mathrm{m}$-xylene relevant for this work.

\section{Crystal structure and calculated dynamics}

The crystal structure of m-xylene was solved recently using the high-resolution powder diffractometer HRPD at the ISIS Facility, $\mathrm{UK}^{5}$ using a deuterated sample. The space group is Pbca with eight molecules per unit cell, $Z=8$. The aromatic ring is essentially planar exhibiting slight angular deformations at the sides of the substituents. The rotational orientation of the methyl groups with respect to the aromatic ring is different: One methyl $\mathrm{D}$ atom is essentially perpendicular to the plane whereas in the other group a deviation of $19^{\circ}$ from this perpendicular direction is present. Structural properties of the crystal are given in Table I.

For an isolated m-xylene molecule both methyl groups undergo nearly free rotation rotation. However, the crystal structure imposes a hindrance for methyl reorientation. By using the crystal structure and TPP the tunneling, firstlibrational, activation energies as well as the potential parameters have been calculated ${ }^{25}$ and are summarized in Table II.

\section{THEORY}

The single particle model $\left(\mathrm{SPM}^{6}\right)$, which describes the dynamics of many molecular crystals containing, e.g., $\mathrm{CH}_{3}$ groups, assumes an isolated group whose reorientations are hindered via its interaction with its environment. Often these 
TABLE I. Structural properties of the deuterated m-xylene (Ref. 5). Atomic positions of atoms belonging to the respective methyl group and the assigned tunneling frequency are shown as well.

\begin{tabular}{|c|c|c|c|c|c|}
\hline & \multicolumn{5}{|c|}{$\begin{array}{c}a=10.1550 \AA \\
b=7.4649 \AA \\
c=16.8841 \AA\end{array}$} \\
\hline & \multicolumn{2}{|r|}{$\begin{array}{c}\text { Space group } \\
Z \\
\text { Geometry } \\
\text { Method }\end{array}$} & \multicolumn{2}{|c|}{$\begin{array}{c}\text { Pbca (No. 61) } \\
8 \\
\text { orthorhombic } \\
\text { Neutron diffraction }\end{array}$} & \multirow[b]{2}{*}{$\hbar \omega_{t}$ in $\mu \mathrm{eV}$} \\
\hline & Atom & $x / a$ & $y / b$ & $z / c$ & \\
\hline \multirow{4}{*}{$\begin{array}{l}\mathrm{CH}_{3} \\
\text { group \#1 }\end{array}$} & $\mathrm{C}(1 \mathrm{M})$ & 0.5817 & 0.2672 & 0.2684 & \multirow{3}{*}{25.15} \\
\hline & $\mathrm{D}(11)$ & 0.5028 & 0.1995 & 0.1540 & \\
\hline & $\mathrm{D}(12)$ & 0.6172 & 0.2341 & 0.1511 & \\
\hline & $\mathrm{D}(13)$ & 0.5636 & 0.4097 & 0.1734 & \multirow{5}{*}{13.92} \\
\hline \multirow{4}{*}{$\begin{array}{l}\mathrm{CH}_{3} \\
\text { group \#2 }\end{array}$} & $C(3 M)$ & 0.3984 & 0.3082 & 0.4594 & \\
\hline & $\mathrm{D}(31)$ & 0.3179 & 0.2143 & 0.4642 & \\
\hline & $\mathrm{D}(32)$ & 0.3558 & 0.4311 & 0.4401 & \\
\hline & $\mathrm{D}(33)$ & 0.4380 & 0.3254 & 0.5180 & \\
\hline
\end{tabular}

reorientations occur about a fixed axis and, in the case of a $\mathrm{CH}_{3}$ group, the potential that hinders reorientation can be expressed as a function of a single angular coordinate. The total potential energy depends on the symmetry of the rotating group $\left(\mathrm{C}_{3}\right)$ and that of the environment. The rotational potential is often represented as the sum of the first two terms of a Fourier expansion

$$
V_{\mathrm{rot}}(\varphi)=V_{0}+\sum_{n=1}^{2} \frac{V_{3 n}}{2}\left[1-\cos \left(3 n \varphi+\alpha_{3 n}\right)\right]
$$

The potential of Eq. (1) determines the rotational excitations. In the SPM approach these excitations are given by the solutions of the Schrödinger equation

$$
\begin{aligned}
& H\left|\Psi_{i}\right\rangle=E_{i}\left|\Psi_{i}\right\rangle, \\
& {\left[-\frac{\hbar^{2}}{2 I} \frac{d^{2}}{d \varphi^{2}}+V_{\text {rot }}(\varphi)\right] \Psi_{i}=E_{i} \Psi_{i} .}
\end{aligned}
$$

$I$ is the molecular moment of inertia and $\hbar^{2} /(2 I)$ the rotational constant $B$ having a value of $0.655 \mathrm{meV}$ for a methyl group. In order to obtain the energy levels it is sufficient to consider space coordinates. The space wave functions are expanded into free rotor functions $|m\rangle=\exp (\operatorname{im} \varphi)$ according to $\Upsilon_{i}=\Sigma_{m} \alpha_{m}|m\rangle$ and the eigenvalues of the Hamilton matrix $H_{m n}$ are calculated by numerical procedures

$$
\langle m|H| n\rangle=E_{m n}\langle m \mid n\rangle .
$$

Parameters of rotational potentials can be derived from experimentally determined transition energies and by choosing arbitrarily $V_{0}=0 \mathrm{meV}$ and $\alpha_{3}=0^{\circ}$ in Eq. (1). In the following sections two experimental values will be obtained, the

TABLE II. Potential parameters derived by using the crystal structure and transferable pair potentials (Ref. 25).

\begin{tabular}{lcccc}
\hline \hline $\begin{array}{l}\hbar \omega_{t}^{\text {calc }} \\
{[\mu \mathrm{eV}]}\end{array}$ & $\begin{array}{c}E_{01}^{\mathrm{calc}} \\
{[\mathrm{meV}]}\end{array}$ & $\begin{array}{c}E_{a}^{\text {calc }} \\
{[\mathrm{meV}] /[\mathrm{kJ} / \mathrm{mol}]}\end{array}$ & $\begin{array}{c}V_{3}^{\text {calc }} \\
{[\mathrm{meV}]}\end{array}$ & $\begin{array}{c}V_{6}^{\text {calc }} \\
{[\mathrm{meV}]}\end{array}$ \\
\hline 11.6 & 11.9 & $25.9 / 2.5$ & 31.2 & 1.9 \\
24.4 & 10.5 & $17.6 / 1.7$ & 25.2 & -0.9 \\
\hline \hline
\end{tabular}

transition to the first excited librational state and the activation energy. Therefore, only two potential parameters of $V(\varphi)$ can be determined and $\alpha_{6}$ was set to $\alpha_{6}=0^{\circ}$ within the SPM approach.

\section{A. Scattering function for rotational tunneling}

In a neutron scattering experiment one measures a quantity proportional to the scattering function $S(Q, \omega)$. For quantum rotational tunneling among three equivalent sites the scattering function is given by ${ }^{6}$

$$
\begin{aligned}
S(Q, \omega)= & {\left[\frac{5}{9}+\frac{4}{9} j_{0}(Q d)\right] \delta(\omega) } \\
& +\frac{2}{9}\left[1-j_{0}(Q d)\right]\left(\delta\left(\omega-\omega_{t}\right)+\delta\left(\omega+\omega_{t}\right)\right),
\end{aligned}
$$

where $Q$ is the magnitude of the wavevector transfer, $j_{0}(Q d)=\sin (Q d) /(Q d)$ the zeroth-order spherical Bessel function, and $d=1.78 \AA$ the distance between $\mathrm{H}$ atoms of the $\mathrm{CH}_{3}$ group. Equation (3) contains one elastic and two inelastic lines, the latter at energy loss of the neutron, $\hbar \omega_{t}$ and energy gain $-\hbar \omega_{t}$. If a tunneling spectrum contains more than one transition the scattering function is a superposition of single particle spectra weighted with the occurrence probabilities $p_{v}$ of the respective species. Whereas in the isolated molecule both $\mathrm{CH}_{3}$ groups are equivalent they are inequivalent in the crystal and $S(Q, \omega)$ of m-xylene becomes

$$
S(Q, \omega)=p_{1} S_{1}(Q, \omega)+p_{2} S_{2}(Q, \omega),
$$

with $p_{1}=p_{2}=1 / 2$ as derived from the crystallographic structure. Since Eq. (3) is valid only for $T=0 \mathrm{~K}$, the $\delta$-functions are replaced by Lorentzians with a given full width at half maximum (FWHM) $\Gamma$ for $T>0 \mathrm{~K}$. The broadening of the observed tunneling lines with increasing temperature $T$ is described as an Arrhenius law ${ }^{7}$

$$
\Gamma(T)=\Gamma_{0} \cdot \exp \left(-\frac{E_{\Gamma}}{k_{B} T}\right)
$$


To a good approximation the line broadening is caused by the transition to the first librational level at energy $E_{01}$ and thus $E_{\Gamma} \approx E_{01}$. Therefore, $E_{01}$ can be obtained from fitting the experimentally obtained values of $\Gamma(T)$ against $T$ or $1 / T$.

\section{B. Scattering function for quasi-elastic scattering}

At elevated temperatures reorientations can occur when the single particle has enough thermal energy to hop over the barrier. The scattering function $S(Q, \omega)$ for a powder sample with protons jumping between three equivalent sites on a circle with a jump frequency $\nu$ is given by

$$
S(Q, \omega)=A_{0}(Q) \delta(\omega)+A_{1}(Q) \frac{1}{\pi} \frac{\frac{3}{2} \nu}{\left(\frac{3}{2} \nu\right)^{2}+\omega^{2}},
$$

with the pre-factors $A_{0}(Q)=\frac{1}{3}\left[1+2 j_{0}(Q d)\right]$ and $A_{1}(Q)$ $=\frac{2}{3}\left[1-j_{0}(Q d)\right] .^{8}$ Again, $Q$ is the value of the scattering vector and $d$ the jump length, i.e., the proton-proton distance of $d=1.78 \AA$ inside the $\mathrm{CH}_{3}$ group. The width of the Lorentzian in Eq. (5) is given by $\Gamma=(3 / 2) \nu$. Since the proton jumps are thermally activated processes the jump frequency $\nu$ and, therefore, the linewidth $\Gamma$ of the Lorentzian depends on the temperature and shows an Arrhenius behavior, too. The activation energy $E_{a}$ which yields the energy difference between the ground state and the maximum of the methyl rotational potential can be obtained by replacing $E_{\Gamma}$ by $E_{a}$ in Eq. (4). Of course, the prefactor $\Gamma_{0}$ will change accordingly. Due to its two inequivalent methyl groups the reorientation rates result (ideally) in linewidths $\Gamma^{(i)}$ that have different temperature dependences. This specific temperature dependence is used to determine the group specific activation energies $E_{a}^{(i)}$. Again, the experimental scattering function $S(Q, \omega)$ is a superposition of individual scattering functions as mentioned earlier.

\section{EXPERIMENT}

\section{A. Sample preparation}

The sample material is commercially available from the Aldrich company and the chemical purity was better than 99\%. ${ }^{9}$ The boiling point of $\sim 139^{\circ} \mathrm{C}$ did not require any special sample preparation besides standard laboratory preparation procedures. The sample container used at the HFBS spectrometer was a hollow cylinder, ${ }^{10}$ and the one used at the NEAT spectrometer a flat shaped aluminum container. The latter one was oriented under $45^{\circ}$ in the neutron beam. The amount of sample material was chosen to get a scattering probability of $25 \%$.

\section{B. Data processing}

The experimental data obtained were transformed into $S(Q, \omega)$ using standard programmes (Refs. 11 and 12) to derive the relevant physical parameters. The evaluation involved the following steps:

(1) Background subtraction;

(2) determination of the resolution function;

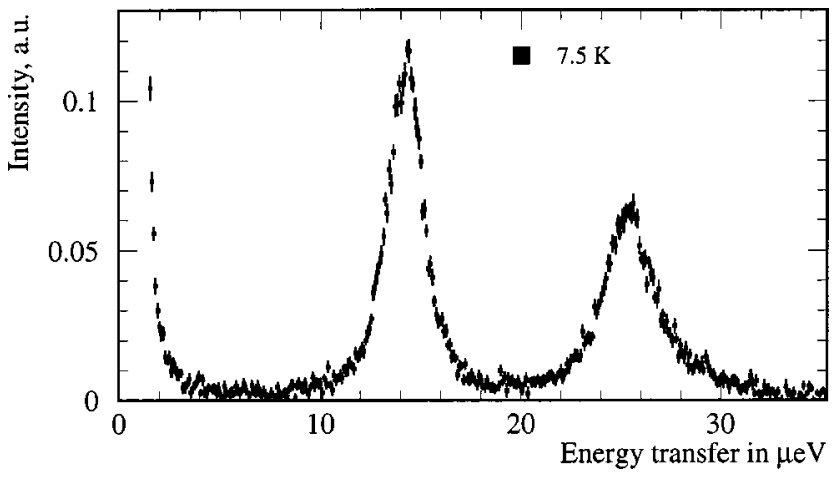

FIG. 1. Tunneling spectrum of m-xylene at 7.5 K. Two peaks are visible at $\hbar \omega_{t}=13.92 \mu \mathrm{eV}$ and $\hbar \omega_{t}=25.15 \mu \mathrm{eV}$.

(3) convolution of the theoretical spectrum with the resolution function;

(4) fit and comparison with the measured spectra.

\section{Inelastic scattering}

After a first high-resolution experiment at the IRIS spectrometer of the ISIS spallation source, which showed for the first time two tunneling systems and a set of measurements at the Jülich backscattering spectrometer the final inelastic neutron spectrum was obtained using the high resolution backscattering spectrometer HFBS at the National Institute of Standards (NIST) in Gaithersburg, MD, US. The lowest temperature of $7.5 \mathrm{~K}$ was achieved using a closed-cyclerefrigerator. The energy transfer varied within $\pm 36 \mu \mathrm{eV}$ with an energy resolution of $\delta \mathrm{E}=1.01 \mu \mathrm{eV}$ at the elastic peak at an incident wavelength of $\lambda=6.27 \AA$. The spectrum yields two sets of tunneling lines at $\hbar \omega_{t}= \pm 13.92 \mu \mathrm{eV}$ and $\hbar \omega_{t}$ $= \pm 25.15 \mu \mathrm{eV}$, see Fig. 1. The integrated intensities of the tunneling peaks are the same, verifying the crystallographic occurrence probabilities of $p_{1}=p_{2}=1 / 2$. To obtain values for the librational transitions, the temperature dependence of the FWHM of the observed lines was used, see Fig. 2.

The widths of the two tunneling lines were obtained by fitting the individual line widths at 11 different temperatures within the range of $7.5 \mathrm{~K} \leqslant T \leqslant 24 \mathrm{~K}$. A simple Arrhenius behavior, as given by Eq. (4), does not satisfactorily describe

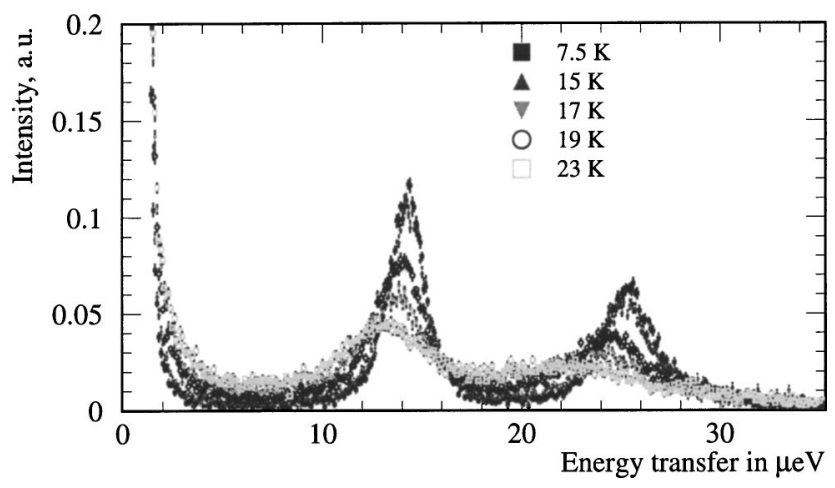

FIG. 2. Temperature-dependent line broadening and positional shift of the tunneling lines of m-xylene. 


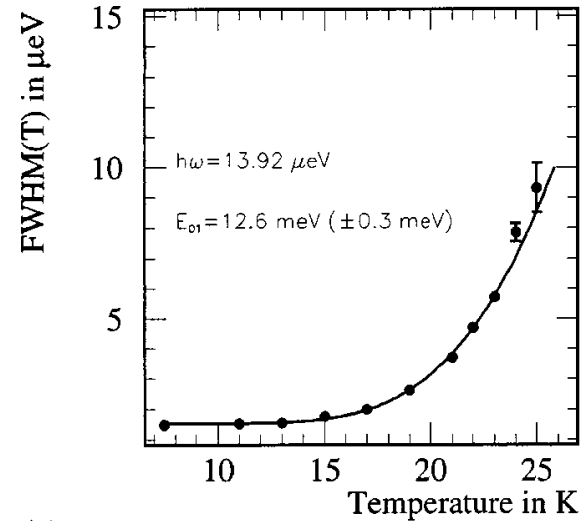

(a)

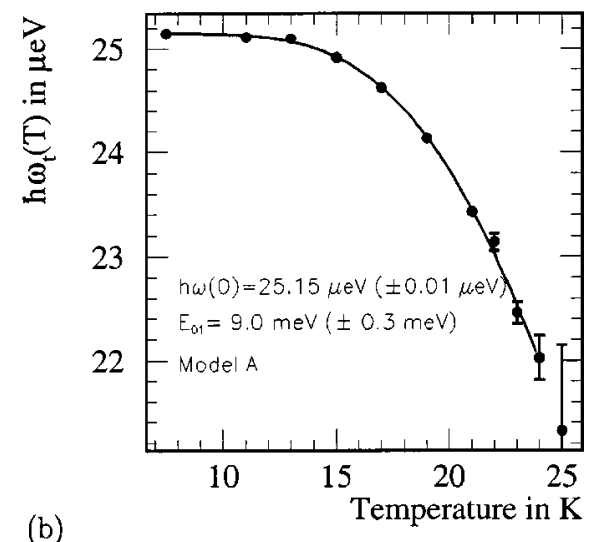

FIG. 3. Temperature-dependent FWHM line broadening of the two tunneling lines in $\mathrm{m}$-xylene. The data were collected in a temperature range of $7.5 \mathrm{~K} \leqslant T \leqslant 29 \mathrm{~K}$. The fitted range was chosen to be between 7.5 and $24 \mathrm{~K}$.

the temperature dependence of the linewidth. A zerotemperature nonzero linewidth is necessary, thus Eq. (4) becomes

$$
\Gamma(T)=\Gamma_{0} \cdot \exp \left(-\frac{E_{\Gamma}}{k_{B} T}\right)+C .
$$

Using Eq. (6) one can obtain values for $\mathrm{E}_{\Gamma}^{(i)}$ by fitting $\Gamma(T)$ as shown in Fig. 3. The parameters obtained from a leastsquare fit are shown in Table III.

The introduction of the parameter $C$ in Eq. (6) may be interpreted as a hint of phenomena such as coupling between the librations of neighboring proton groups. Punkkinen introduced a model ${ }^{13}$ taking into account coupling between protons of neighboring methyl groups, whose extension to the SPM approach results in Eq. (6). Note that in the limit of $C \ll \Gamma_{0} \cdot \exp \left(-E_{\Gamma} / k_{B} T\right)$ the expression in Eq. (6) becomes the

TABLE III. Parameters obtained using the modified Arrhenius model to describe the temperature-dependent line broadening of the tunneling peaks.

\begin{tabular}{cccc}
\hline \hline $\begin{array}{c}\hbar \omega_{t}^{\exp } \\
{[\mu \mathrm{eV}]}\end{array}$ & $\begin{array}{c}E_{01}^{\exp } \\
{[\mathrm{meV}]}\end{array}$ & $\begin{array}{c}\Gamma_{0} \\
{[\mu \mathrm{eV}]}\end{array}$ & $\begin{array}{c}C \\
{[\mu \mathrm{eV}]}\end{array}$ \\
\hline $13.92( \pm 0.01)$ & $12.6( \pm 0.3)_{\mathrm{FWHM}}$ & $2.4( \pm 0.4)$ & $1.5( \pm 0.01)$ \\
$25.15( \pm 0.01)$ & $10.6( \pm 0.4)_{\mathrm{FWHM}}$ & $1.5( \pm 0.3)$ & $2.8( \pm 0.03)$ \\
\hline \hline
\end{tabular}
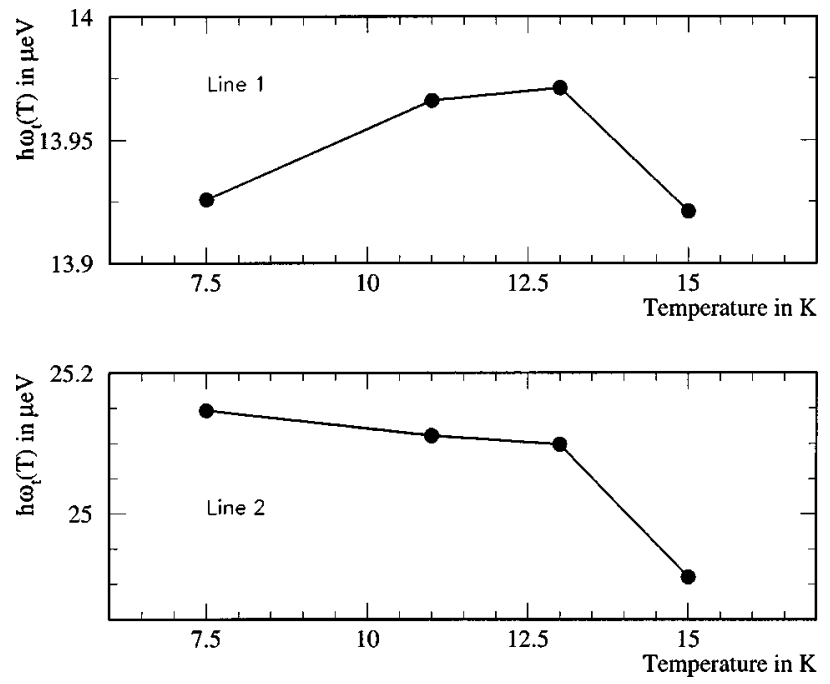

FIG. 4. Temperature-dependent shift of the observed tunneling lines up to $15 \mathrm{~K}$. The position of the $13.92 \mu \mathrm{eV}$ peak increases with increasing temperature up to $13 \mathrm{~K}$ (Line 1, top). Above $13 \mathrm{~K}$ the expected behavior takes place. The $25.15 \mu \mathrm{eV}$ tunnel peak shows expected temperature dependence (Line 2, bottom). The lines are guides to the eye.

"classical" Arrhenius form of Eq. (4). The presence of a linewidth at low temperatures may also be indicative of some disorder, which will be discussed later on.

The temperature-dependent shift of the center of the tunneling lines can provide additional information on the microscopic dynamics. The line at $25.15 \mu \mathrm{eV}$ shifts to lower energy transfers with increasing temperature and shows an "expected" behavior. However, the line at $13.92 \mu \mathrm{eV}$ slightly shifts to higher energy transfers as the temperature increases to $13 \mathrm{~K}$ before decreasing with subsequent temperature increase, see Fig. 4. A similar behavior with increasing temperature was also observed, e.g., in $\mathrm{CH}_{3} \mathrm{I},{ }^{14}$ $\left(\mathrm{NH}_{4}\right)_{2} \mathrm{SnBr}_{6},{ }^{15}$ or $\left(\mathrm{NH}_{4}\right)_{2} \mathrm{ZnCl}_{4} \cdot{ }^{16}$

\section{Coupling to phonons}

Although a theoretical description of the line shift is more complicated, theory predicts an Arrhenius behavior as well. ${ }^{7}$ By using a modified exponential similar to Eq. (6)

$$
\hbar \omega_{t}(T)=\hbar \omega_{t}(0)\left[1-A^{\sin } \cdot \exp \left(-\frac{E_{01}^{\sin }}{k_{B} T}\right)\right] .
$$

(Model A) the temperature-dependent positions of the peak with a low-temperature value of $25.15 \mu \mathrm{eV}$ can be well described. Applying the same model to the peak at $13.92 \mu \mathrm{eV}$ does not fit the data with the same quality. One possible explanation for this unusual temperature-dependence is orientational hindering due to intramolecular forces. However, $a b$ initio calculation showed ${ }^{25}$ that this effect is negligible. Another possibility is that a coupling exists between the tunneling rotor to low-frequency phonons, modulating the single-particle potential, resulting in an increase of the tunnel splitting with increasing temperature. A simplified Hamiltonian simulating the phonons as a single harmonic oscillator with dispersionless Einstein modes is given by 
TABLE IV. Parameters obtained from fitting the experimental values of the temperature-dependent line shift according to Models A and B, see text.

\begin{tabular}{cccccrc}
\hline \hline Model & $\begin{array}{c}\hbar \omega_{t}(0) \\
{[\mu \mathrm{eV}]}\end{array}$ & $A^{\cos }$ & $A^{\text {sin }}$ & $\begin{array}{c}E^{\cos } \\
{[\mathrm{meV}]}\end{array}$ & $\begin{array}{c}E_{01}^{\text {sin }} \\
{[\mathrm{meV}]}\end{array}$ & $\chi^{2}$ \\
\hline A & $13.96( \pm 0.01)$ & & 60.6 & & $13.3( \pm 0.3)$ & 4.2 \\
B & $13.88( \pm 0.02)$ & 0.03 & 32.9 & $0.3( \pm 0.1)$ & $11.1( \pm 0.4)$ & 2.8 \\
A & $25.15( \pm 0.01)$ & & 9.9 & & $9.0( \pm 0.3)$ & 0.9 \\
B & $25.14( \pm 0.02)$ & 0.29 & 8.7 & $6.0( \pm 0.5)$ & $8.9( \pm 0.8)$ & 1.0 \\
\hline \hline
\end{tabular}

$$
\begin{aligned}
H= & \frac{L_{\varphi}^{2}}{2 \Theta}+V_{3} \cos 3 \varphi+\frac{P^{2}}{2 m}+\frac{m}{2} \omega^{2} x^{2} \\
& +\sqrt{\frac{2 m \omega}{\hbar}} x\left\{g_{\cos } \cos 3 \varphi+g_{\sin } \sin 3 \varphi\right\} .
\end{aligned}
$$

The Hamiltonian in Eq. (8) includes a cos-term, which modulates the amplitude and a sin-term, which shifts the rotational potential. The numerical solution shows that the tunneling frequencies of two adjacent oscillator levels have approximately a constant ratio $\hbar \omega_{t}(n+1) / \hbar \omega_{t}(n)=1$ $+A^{\cos }$ with $A^{\cos }$ being a coupling parameter to phonons. The following empirical equation for describing the temperature dependence of the tunneling lines, based on the Hamiltonian in Eq. (8), is applied (Model B): $:^{14}$

$$
\begin{aligned}
\hbar \omega_{t}(T)= & \hbar \omega_{t}(0)\left[\frac{1-\exp \left(-E^{\cos } / k_{B} T\right)}{1-\left(1+A^{\cos }\right) \exp \left(-E^{\cos } / k_{B} T\right)}\right. \\
& \left.-A^{\sin } \exp \left(-E_{01}^{\sin } / k_{B} T\right)\right] .
\end{aligned}
$$

Here the first term in brackets encapsulates the effects of phonons modulating the potential barrier where $E^{\cos }$ is an effective phonon energy. The second term represents the sincoupling resulting in a shift of the tunneling frequency to lower values if the temperature increases. The Arrhenius behavior is thus obtained for high temperatures. Parameters obtained by fitting the two models to the experimental data are given in Table IV.

Looking at Table IV, statistical considerations would favor Model $B$ as the best description for the $13.92 \mu \mathrm{eV}$ tunneling line according to $\chi_{\text {Model B }}^{2}<\chi_{\text {Model A }}^{2}$. The same consideration would favor Model A for the $25.15 \mu \mathrm{eV}$ tunneling line because of $\chi_{\text {Model B }}^{2}>\chi_{\text {Model } A}^{2}$. Consistent with the theory is the prediction $E_{01}^{\mathrm{sin}} \leqslant E_{\Gamma} \approx E_{01}$ based on statistical considerations. The value of $E_{01}^{\mathrm{sin}}=13.26 \mathrm{meV}$ obtained by using Model A contradicts theory since the value derived from the line broadening is $E_{01}=12.6 \mathrm{meV}$, the assumption of coupling. The fit according to both models is shown in Fig. 5.

A third possible explanation for the unusual temperaturedependence of the $13.92 \mu \mathrm{eV}$ peak is a temperaturedependent expansion-contraction of the lattice parameters. Since the shift occurs for one tunneling peak only, there should be an axis-dependent expansion-contraction, which would influence the local environment felt by the methyl group whose tunnel-splitting at low-temperature is 13.92 $\mu \mathrm{eV}$. A refined temperature-dependent determination of the lattice parameters shows some anisotropic variation of the lattice parameters. ${ }^{17}$ The parameters $a$ and $b$ slightly increase whereas $c$ slightly decreases with higher temperatures. However, in the temperature range of $7.5 \mathrm{~K} \leqslant T \leqslant 15 \mathrm{~K}$ these variations are small and the model of coupling to phonons seems to be an appropriate choice.

\section{E. Line broadening}

As mentioned earlier the structure determination of m-xylene reveals two inequivalent $\mathrm{CH}_{3}$ groups with occurrence probabilities of $p_{1}=p_{2}=1 / 2$. In a neutron scattering experiment this leads to identical integrated intensities of the observed inelastic lines. Normally, if one assumes no orientational disorder, all parameters of the rotational potential are $\delta$-distributed implying that, e.g., crystallographic equivalent methyl groups see the same environment, independent of its

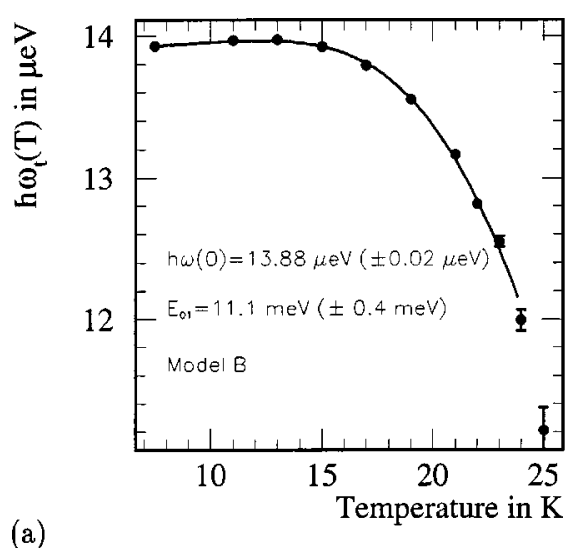

(a)

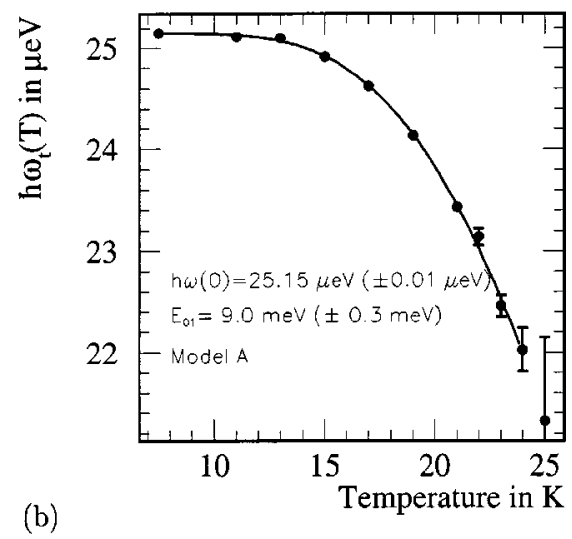

FIG. 5. Least-squares fit of the temperature-dependent shift of the tunneling lines. 
location in the crystal. Under this condition Eqs. (1) and (2) allow one to extract the potential parameters. Thus if the occurrence probabilities are the same and the potential distributions are $\delta$-functions, then both the integrated intensities and the spectral peak heights should be the same.

However, the low temperature inelastic neutron spectrum, Fig. 1, shows that both peaks have the same integrated intensity but different peak heights. Since the instrumental resolution is constant over the whole energy range, an intrinsic width, which can be seen easily for the $25.15 \mu \mathrm{eV}$ tunneling peak, must lead to a decrease of the peak height. The assumption of constant instrumental resolution together with the model applied in Sec. III C implies that this intrinsic line width is of a physical nature.

\section{Coupling of librating methyl groups}

Since from statistics follows that the temperature dependence of the tunneling line at $13.92 \mu \mathrm{eV}$ can be described in terms of phonon coupling the transition at $25.15 \mu \mathrm{eV}$ may be related to coupled methyl libration as discussed by Punkinnen. A simple Hamiltonian that can be used to couple two methyl groups to each other is given by

$$
H=H_{1}+H_{2}+W_{12},
$$

with the single particle terms

$$
H_{i}=-B_{i} \frac{\partial^{2}}{\partial \varphi_{i}^{2}}+\frac{V_{3 i}}{2}\left(1-\cos 3 \varphi_{i}\right), \quad i=1,2,
$$

where $B_{i}$ denotes the rotational constant of the methyl groups. If the coupling depends on the differences of orientations only $W_{12}$ can be expressed as

$$
W_{12}=W_{3} \cos \left(3 \varphi_{1}-3 \varphi_{2}\right) \text {. }
$$

Calculations show ${ }^{19}$ that for crystallographically equivalent and identical particles, $V_{3}=V_{31}=V_{32}$ and $B_{1}=B_{2}$, the librational ground state consists of four tunneling sublevels and transitions between $A A \rightarrow A E, A A \rightarrow E_{a} E_{b}, A A \rightarrow E_{a} E_{a}$ and $E_{a} E_{a} \rightarrow E_{a} E_{b}$ are possible.

A fit of the experimental data, in particular the second peak at higher energy transfers, Fig. 6, yields a main tunneling transition, representing the $A A \rightarrow A E$ transition, of $\hbar \omega_{t}$ $=25.67 \mu \mathrm{eV}$. From the peak positions of the Lorentzians used to fit the experimental spectrum we obtain $\delta \hbar \omega_{t}$ $=-0.97 \mu \mathrm{eV}$. The diagonalization of the Hamiltonian given in Eq. (10) yields transitions for the combination of $\left(V_{3}, W_{3}\right)=(14.73 \mathrm{meV},-6.07 \mathrm{meV})$, which are shown in Table V. The mean value of the tunneling transition is $\overline{\hbar \omega_{t}}$ $=25.13 \mu \mathrm{eV}$ and the experimental peak at about $25.15 \mu \mathrm{eV}$ could be interpreted as being an average value. Diagonalizing the model hamiltonian allows to calculate, e.g., the activation energy, which is taken as the distance between the ground-state energy and the saddle point of the potential surface $V\left(\varphi_{1}, \varphi_{2}\right)$. For the derived $\left(V_{3}, W_{3}\right)$ combination we obtain a value of $E_{a}=15.7 \mathrm{meV}$ which is very close to and consistent with the experimental value obtained from quasielastic experiments described in Sec. IIIF. Contrary to the SPM the coupling leads to a more complex scheme regarding the transitions to excited librational states. Here, transitions between different states of symmetry in the range of $\sim 7.8$

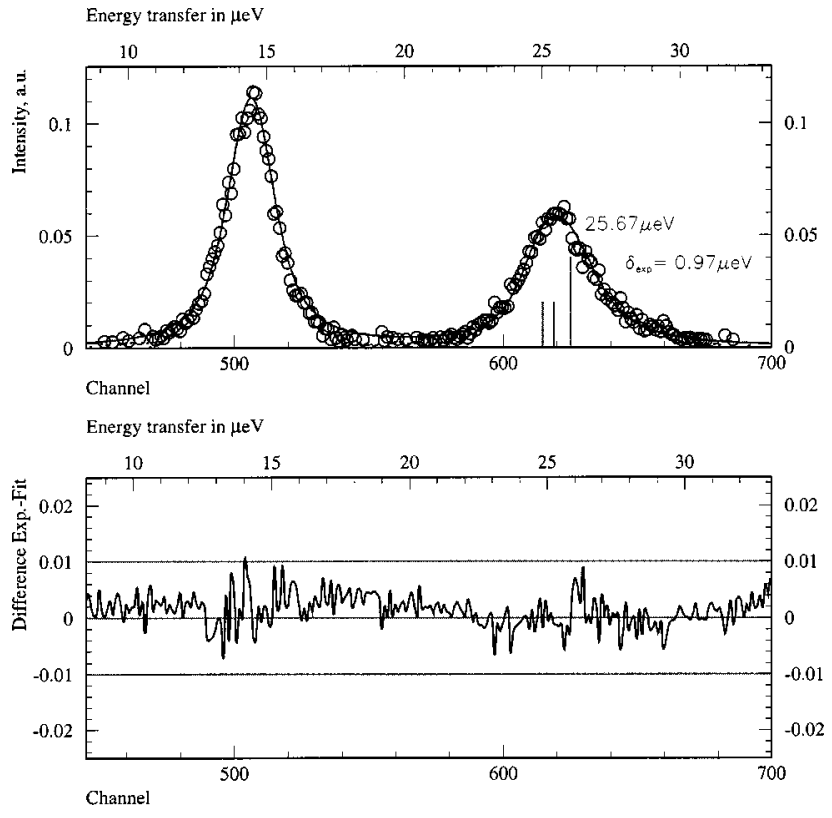

FIG. 6. Fit of the experimental data at $7.5 \mathrm{~K}$ with a model that couples the libration of two equivalent methyl groups. There is reasonable agreement between the data and the fit.

meV up to $\sim 12.4 \mathrm{meV}$ are calculated. By assuming dispersion of librational modes within the Brillouin zone it can be understood that no distinct librational peaks were observed in an earlier experiment. ${ }^{18}$ Furthermore, the results are consistent with Ref. 19 in which it is explained how a coupling term $W_{3}<0$ counteracts the single particle potential $V_{3}$, thus making the effective potential harder.

It should be mentioned as well that the tunneling peak at lower energy transfers is well described as a single peak only with $\delta \hbar \omega_{t}=0 \mu \mathrm{eV}$ indicating $W_{3}=0 \mathrm{meV}$. Again, the crystal structure shows that the methyl groups that see the potential causing this tunneling transition are arranged almost perpendicular to each other. This geometry leads to a much weaker (not-observable) coupling at similar intermolecular distances contrary to the almost parallel and in-plane oriented methyl groups.

\section{Rotational potential distribution}

Of course, coupling of motions may not be the only explanation. A second possibility, briefly described in the following, assumes that a random distribution of different neighborhoods may be the reason for the observed line

TABLE V. Calculated transition between the ground-state sublevels for the experimental tunneling peak at higher energy transfers.

\begin{tabular}{cc}
\hline \hline Symmetry & Transition in $\mu \mathrm{eV}$ \\
\hline$A A \rightarrow A E$ & 25.67 \\
$A A \rightarrow E_{a} E_{b}$ & 24.70 \\
$A A \rightarrow E_{a} E_{a}$ & 25.03 \\
$E_{a} E_{b} \rightarrow E_{a} E_{a}$ & 0.33 \\
\hline \hline
\end{tabular}




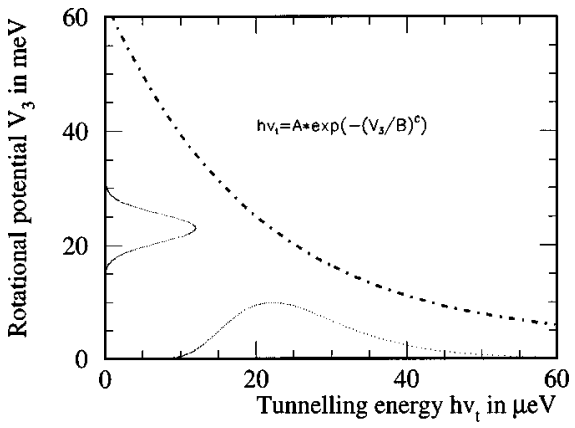

FIG. 7. Line shape of an arbitrary tunneling line depending on the normal distributed $V_{3}$ part of the rotational potential in case of orientational disorder. Here an exponential dependence of the tunneling energy on the potential has been used for illustration.

shapes. A simple approach constrains the rotational potential to the $V_{3}$ part of Eq. (1) and assumes this part is to be normal distributed, e.g., ${ }^{20,21}$

$$
g\left(V\left(\hbar \omega_{t}\right)\right)=\frac{1}{(2 \pi)^{1 / 2} \cdot \delta V_{3}} \exp \left(-\frac{1}{2}\left(\frac{V\left(\hbar \omega_{t}\right)-V_{3}}{\delta V_{3}}\right)^{2}\right),
$$

and the distribution of the tunneling intensities can be obtained from

$$
I\left(\hbar \omega_{t}\right)=-g\left(V\left(\hbar \omega_{t}\right)\right)\left(\frac{d\left(\hbar \omega_{t}(V)\right)}{d V}\right)^{-1} .
$$

The coordinate transformation is schematically shown in Fig. 7 and reflects the asymmetry of the observed tunneling line due to its exponential dependence on the rotational potential.

Parameters of the potential distributions fitting the lowtemperature tunneling spectra are given in Table VI and shown in Fig. 8. The assumption of a Gaussian distribution of potentials describes the spectrum reasonably well, especially with regards to the asymmetry of the tunneling peak at $25.15 \mu \mathrm{eV}$ but is not as good as the fit assuming the coupling.

The relative disorder is similar in magnitude for both lines. However, the asymmetry of the line shape is much less apparent for the lower energy tunnel splitting than it is for the higher one. As suggested in Fig. 7, the line shape for a smaller barrier appears more symmetric than hat due to a higher barrier. Since no method is so sensitive to disorder like rotational tunneling spectroscopy, see, e.g. Ref. 22, the observed line broadening could indicate the presence of some long-range misorientations of the crystal lattice but no disorder was reported during the structure determination (besides the angular deformations mentioned earlier), which would endorse this model.

TABLE VI. Potential distributions derived from the tunneling positions and line-widths.

\begin{tabular}{ccccc}
\hline \hline $\begin{array}{c}\hbar \omega_{t} \\
{[\mu \mathrm{eV}]}\end{array}$ & $\begin{array}{c}\mathrm{V}_{3} \\
{[\mathrm{meV}]}\end{array}$ & $\begin{array}{c}\delta \mathrm{V}_{3} \\
{[\mathrm{meV}]}\end{array}$ & $\delta \mathrm{V}_{3} / \mathrm{V}_{3}$ & $\begin{array}{c}\text { Intensity } \\
{[\mathrm{a} . \mathrm{u}]}\end{array}$ \\
\hline 14.2 & 27.5 & 0.5 & 0.02 & 1.0 \\
25.4 & 22.9 & 0.4 & 0.03 & 1.0 \\
\hline \hline
\end{tabular}
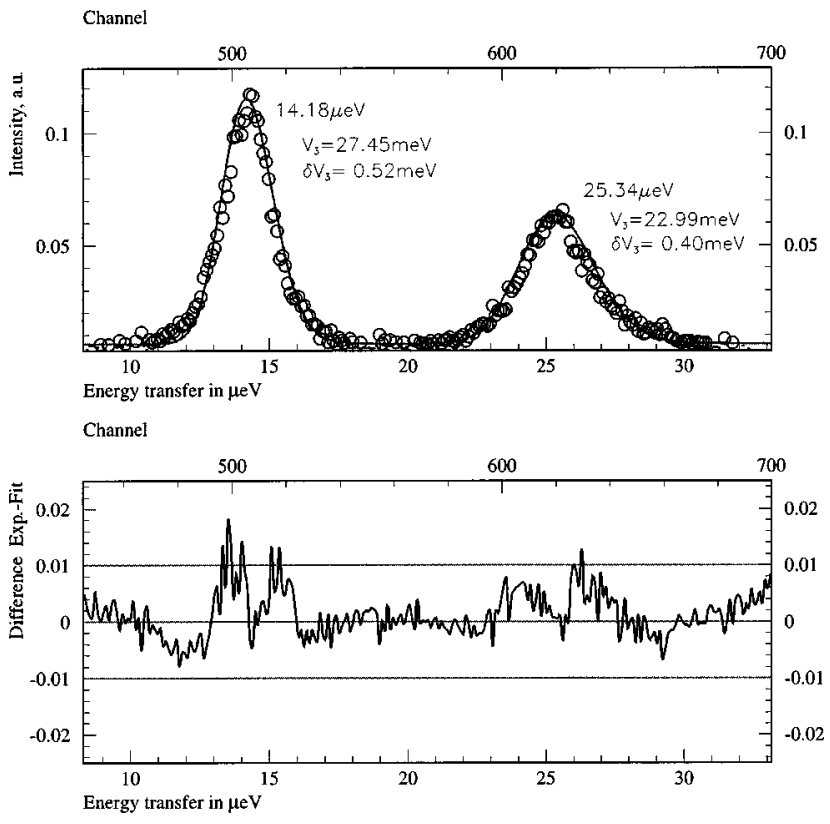

FIG. 8. Fit of the tunneling intensity distributions according to Eq. (14).

\section{F. Quasi-elastic scattering}

In order to obtain values for the activation energy eight quasi-elastic spectra were collected in the temperature range of $40.42 \mathrm{~K} \leqslant T \leqslant 90.28 \mathrm{~K}$ using the multi disc-chopper spectrometer NEAT at the Hahn-Meitner-Institute (HMI), Berlin, GER. The incident neutron wavelength was chosen to be $\lambda=7.0 \AA$ corresponding to an energy resolution of $\delta \mathrm{E}=50$ $\mu \mathrm{eV}$ at the elastic line. The energy transfer range used for fitting the data varied from -0.4 to $0.4 \mathrm{meV}$.

Again, the collected data were transformed into $S(Q, \omega)$ using standard procedures. The experimental spectra and their broadening, see Fig. 9, were analyzed with respect to the temperature dependent FWHM of the Lorentzians according to Eq. (4). During the data processing the relative intensities of the two Lorentzians were fixed at a ratio of $1: 1$ according to their occurrence probability $p_{1}=p_{2}$.

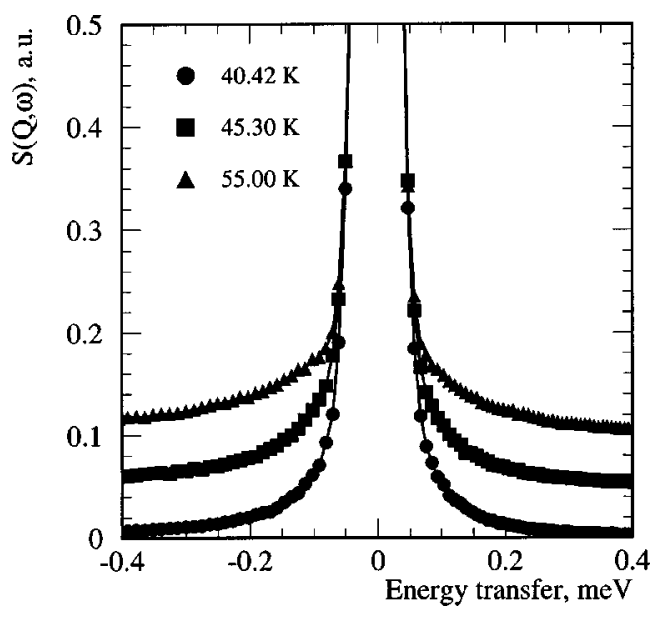

FIG. 9. Quasi-elastic spectrum of m-xylene and the temperature dependant line broadening, shown at three different temperatures. 

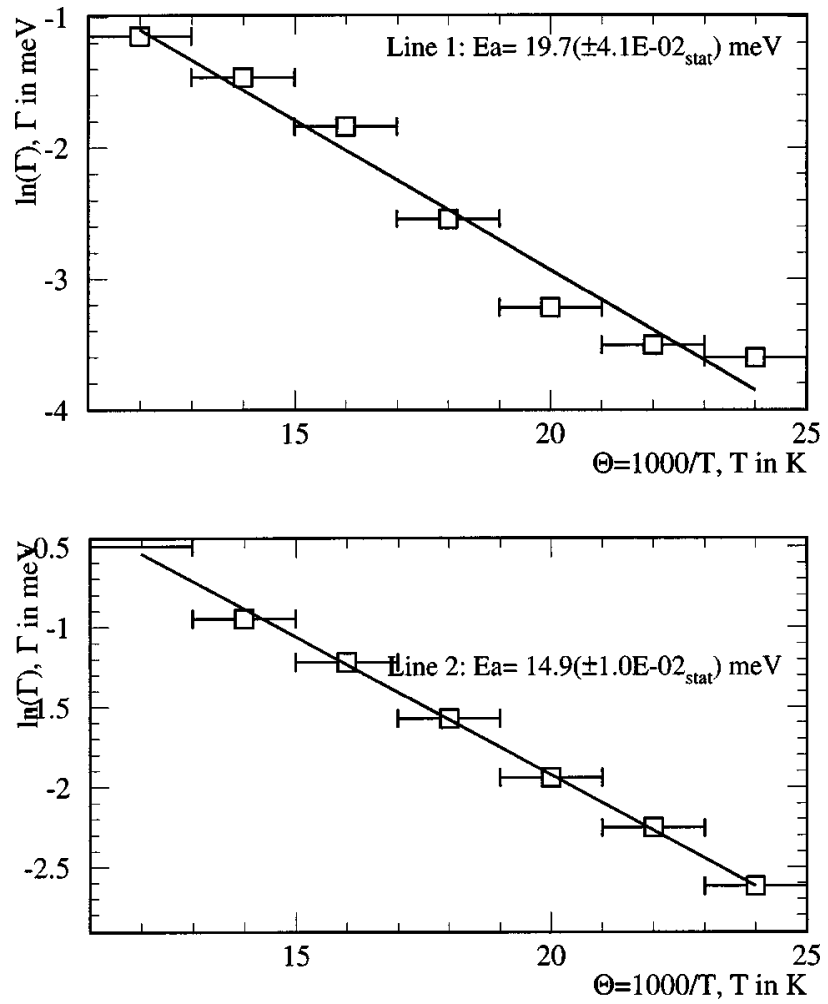

FIG. 10. Arrhenius plot of the temperature dependence of the widths of the observed quasi-elastic lines.

The widths of the Lorentzians at different temperatures show an Arrhenius behavior according to Eq. (4) with $E_{\Gamma}$ replaced by $E_{a}$. For both lines a least-squares fit to the linewidths was performed as shown in Fig. 10. The pre-factors $\Gamma_{0}$ were calculated to be 4.6 and $5.1 \mathrm{meV}$ and are consistent with the attempt frequency found elsewhere for methyl groups. From the least-squares fit, activation energies of $E_{a}^{1}$ $=19.7 \mathrm{meV}$ and $E_{a}^{2}=14.9 \mathrm{meV}$ were obtained.

\section{PARAMETERS OF THE ROTATIONAL POTENTIAL WITHIN SPM}

Besides the hints of coupling the results of the inelastic and quasi-elastic neutron scattering experiments are used to derive two parameters of the Fourier-expansion of the potential given by Eq. (1). By choosing arbitrarily $\alpha_{3}=0^{\circ}, V_{0}$ $=0 \mathrm{meV}$ and using a rotational constant of $B=0.655 \mathrm{meV}$ for the respective $\mathrm{CH}_{3}$ group, the values displayed in Table VII were obtained. The SPM model used to describe the dynamics of the methyl group rotations represent the experimentally obtained values for the librational transition as well as the activation energies quite accurately if the SPM results are understood as mean values, in particular regarding the tunneling transition at higher energy transfers.

Another representation of the rotational potential is possible by defining the parameters $V S=\left|V_{3}\right|+\left|V_{6}\right|$ as well as $\delta=V_{3} / V S$. VS describes the strength of the potential and $\delta$ characterizes the deviation from a pure threefold potential. Values of these parameters are given in Table VII as well. As can be seen, the deviation from a pure threefold cosine potential is small and may confirm the procedure used in Secs. IIIE 1 and IIIE 2 in which only the $V_{3}$ component of the potentials were taken into account.

\section{ASSIGNMENT}

In the crystalline state, rotational tunneling frequencies of methyl groups are very sensitive probes of the local environment, as already mentioned. Thus obtaining different tunneling frequencies means that different methyl groups are either coupled or see different local environments. An unambiguous assignment of a tunneling frequency to the respective methyl group can be done by, e.g., following the relative intensities of the tunneling peaks as a function of momentum transfer along a particular crystal plane. This technique requires not only the knowledge of the crystal structure but a single crystal as well. By using single crystal experiments methyl groups were assigned to tunneling frequencies in, e.g., 2,6-dimethylpyrazine ${ }^{2,23}$ or tribromomesitylene. ${ }^{24} \mathrm{An}$ assignment of $\mathrm{CH}_{3}$ groups to tunneling frequencies using a powder sample is possible if the crystal structure is known and the dynamics can be modeled by, e.g., using harmonic approximation in combination with transferable pair potentials or $a b$ initio and molecular-mechanics calculations in reasonable agreement with the experiment.

By combining the experimental results with the information given in Ref. 25 it is possible to assign the observed tunneling lines to the crystallographic $\mathrm{CH}_{3}$ group. The assignment is shown in Fig. 11.

\section{CONCLUSION}

The rotational dynamics of the methyl groups of $\mathrm{m}$-xylene has been investigated by using incoherent inelastic and quasi-elastic neutron scattering. The high-resolution experiment, using the HFBS spectrometer at NIST, showed two tunneling lines, with mean peak positions at 13.92 and 25.15 $\mu \mathrm{eV}$, and their relative integrated intensities agree with the crystallographic results predicting methyl groups of equal

TABLE VII. Calculated rotational parameters based on the observed tunneling energies of $m$-xylene and their temperature-dependent behavior.

\begin{tabular}{ccccccccc}
\hline \hline $\begin{array}{c}\hbar \omega_{t}^{\text {exp }} \\
{[\mu \mathrm{eV}]}\end{array}$ & \begin{tabular}{c}
$E_{01}^{\text {exp }}[\mathrm{meV}]$ \\
\hline 13.92
\end{tabular} & $\begin{array}{c}E_{a}^{\text {exp }} \\
{[\mathrm{meV}]}\end{array}$ & $\begin{array}{c}V_{3}^{\text {spm }} \\
{[\mathrm{meV}]}\end{array}$ & $\begin{array}{c}V_{6}^{\mathrm{spm}} \\
{[\mathrm{meV}]}\end{array}$ & $\begin{array}{c}E_{01}^{\mathrm{spm}} \\
{[\mathrm{meV}]}\end{array}$ & $\begin{array}{c}E_{a}^{\mathrm{spm}} \\
{[\mathrm{meV}]}\end{array}$ & $\begin{array}{c}V S \\
{[\mathrm{meV}]}\end{array}$ & $\delta$ \\
\hline 25.15 & $\begin{array}{c}11.6_{\text {FWHM }} \\
10.6_{\text {FWHM }} \\
9.0_{\text {shift }}\end{array}$ & 19.7 & 26.2 & 2.3 & 11.6 & 19.7 & 28.6 & 0.92 \\
& 14.9 & 21.2 & 3.4 & 10.6 & 14.9 & 24.6 & 0.86 \\
\hline \hline
\end{tabular}




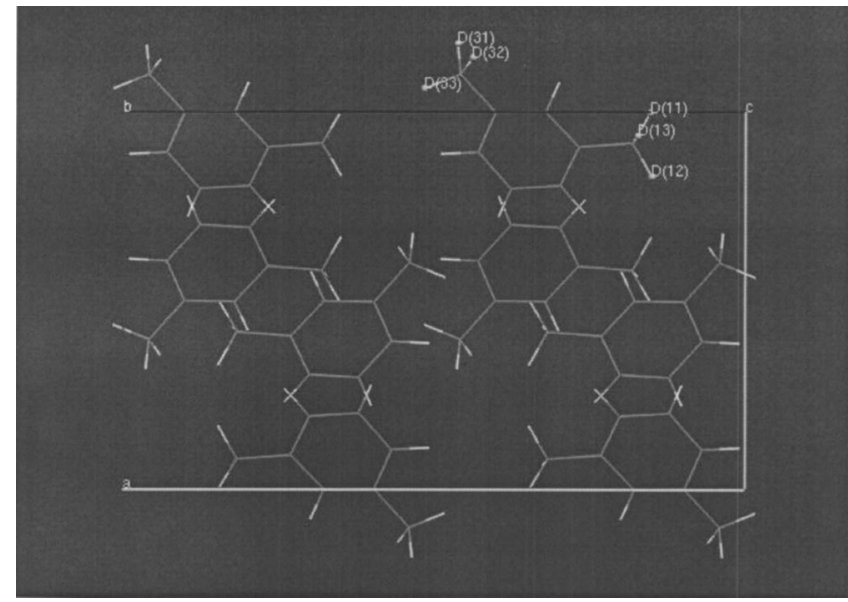

FIG. 11. Unit cell of m-xylene, view along the $b$ axis. The $\mathrm{CH}_{3}$ group assigned to the tunneling peak at $25.15 \mu \mathrm{eV}$ is marked " $\mathrm{D}(11)-\mathrm{D}(12)$ $\mathrm{D}(13)$, , the group assigned to the tunneling peak at $13.92 \mu \mathrm{eV}$ is marked "D(31)-D(32)-D(33)."

occurrence probabilities. The quasi-elastic experiment using the NEAT spectrometer the HMI yielded two activation energies, 19.7 and $14.9 \mathrm{meV}$.

The combination of tunneling, librational, and activation energies allow one to estimate three- and sixfold potential terms. The SPM describes very accurately the experimentally obtained values for the tunnel splittings, first librational and activation energies, which were obtained from the $T$-dependence of the tunneling transitions. However, since there are experimental hints of coupling, the values should be understood as mean values in particular those which are obtained for the low-temperature tunneling peak at $25.15 \mu \mathrm{eV}$.

The unusual temperature dependence of the position of the tunneling peak at $13.92 \mu \mathrm{eV}$ can be understood by coupling the rotation of the methyl group to low energy lattice phonons. The idea of coupling is further endorsed by the modified Arrhenius behavior used to describe the temperature dependencies of the high-resolution data.

For the tunneling line at $25.15 \mu \mathrm{eV}$ the assumption of coupling of the methyl rotation to neighboring groups describes the asymmetry and width of the peak reasonably well. The average of the tunneling transition assuming a $\left(V_{3}, W_{3}\right)$ coupling is $25.13 \mu \mathrm{eV}$. At a first glance, the SPM gives reasonable results with respect to the average values because not only the strength of the coupling is relatively small but the SPM includes a sixfold contribution to the potential. Looking, e.g., at $\epsilon=V_{3} /\left(\left|V_{3}\right|+\left|W_{3}\right|\right)$, which would be similar to the parameter $\delta$ defined within the SPM, may explain that an $\epsilon=0.71$ could be misinterpreted as a $\left(V_{3}, V_{6}\right)$ combination with a relatively high $V_{6}$ contribution and it could be misleading to assume a simple SPM only. Additionally and more importantly, the SPM cannot explain the asymmetry of the tunneling peak at higher energy transfers. The calculated value for the activation energy of $E_{a}$ $=15.7 \mathrm{meV}$ is in good agreement with the experimentally determined value of $E_{a}=14.9 \mathrm{meV}$ endorsing the coupling model. In addition, the more extended excitation scheme regarding the librational transition combined with some dispersion could be an explanation for the lack for librational peaks in the experimental spectrum of the VDOS.

An inhomogeneous distribution of the threefold part of the potential was also considered but since there was neither disorder nor significant distortion of the crystal lattice observed, we believe that coupling is the appropriate model to describe the experimental data.

Finally, by combining the information from previous calculations with the experimental results it was possible to assign the crystallographically identified methyl groups to the experimental tunneling energies.

\section{ACKNOWLEDGMENTS}

One of us (M.P.) would like to thank the ISIS spallation source and the FZJ for the supply of beamtime for test experiments and support during the measurements. Furthermore, O.K. and M.P. would like to thank Dr. H. Grimm (FZJ) for many helpful discussions related to this work. This work is based upon activities supported by the National Science Foundation under Agreement No. DMR-0086210.

${ }^{1}$ M. R. Johnson, M. Prager, H. Grimm, M. A. Neumann, G. J. Kearley, and C. C. Wilson, Chem. Phys. 244, 49 (1999).

${ }^{2}$ B. Nicolai, G. J. Kearley, O. Randl, F. Fillaux, and P. H. Tromsdorff, Physica B 234-236, 76 (1997).

${ }^{3}$ O. Kirstein, M. Prager, M. R. Johnson, and S. F. Parker, J. Chem. Phys. 117, 1313 (2002).

${ }^{4}$ M. Prager, N. Wakabayashi, and M. Monkenbusch, Physica B 202, 252 (1994).

${ }^{5}$ R. M. Ibberson, W. I. F. Shankland, S. Parsons, M. Prager, and K. Shankland, J. Mol. Struct. 524, 121 (2000).

${ }^{6}$ W. Press, Single Particle Rotations in Molecular Crystals, Springer Tracts in Modern Physics (Springer, Berlin, 1981), Vol. 81.

${ }^{7}$ A. Hewson, J. Phys. C 15, 3841 (1985).

${ }^{8}$ M. Beé, Quasielastic Neutron Scattering (Adam Hilger, Bristol, 1998).

${ }^{9}$ Manufacturers are identified in order to provide complete identification of experimental conditions and such identification of experimental conditions is not intended as an endorsement by NIST.

${ }^{10}$ J. Wuttke, Physica B 266, 112 (1999).

${ }^{11}$ See http://www.ncnr.nist.gov $\div$ ave for information related to software package.

${ }^{12}$ TOFSYS is developed and maintained by the Institut für Festkörperforschung, Forschungszentrum Jülich GmbH, Germany.

${ }^{13}$ M. Punkkinen, Phys. Rev. B 21, 54 (1980).

${ }^{14}$ M. Prager, J. Stanislawski, and W. Häusler, J. Chem. Phys. 86, 2563 (1987).

${ }^{15}$ L. P. Ingman, M. Punkkinnen, E. E. Ylinen, and C. Dimitropoulos, Proc. 17th Annu. Conf. Finnish Phys. Soc. 7, 20 (1983).

${ }^{16}$ L. P. Ingman, M. Punkkinnen, A. H. Vuorimäki, and E. E. Ylinen, J. Phys. C 18, 5033 (1985).

${ }^{17}$ R. M. Ibberson (private communication).

${ }^{18}$ M. Prager, R. Hempelmann, H. Langen, and W. Müller-Warmuth, J. Phys.: Condens. Matter 2, 8625 (1990).

${ }^{19}$ W. Häusler and A. Hüller, Z. Phys. B: Condens. Matter 59, 177 (1985).

${ }^{20}$ J. Colmenero, R. Mukhopadyay, A. Alegria, and B. Frick, Phys. Rev. Lett. 80, 2350 (1998).

${ }^{21}$ A. J. Moreno, A. Alegria, J. Colmenero, M. Prager, H. Grimm, and B. Frick, J. Chem. Phys. 115, 8958 (2001).

${ }^{22}$ M. Prager, P. Schiebel, and J. Combet, Chem. Phys. 276, 69 (2002).

${ }^{23}$ B. Nicolaii, E. Kaiser, F. Fillaux, G. J. Kearley, A. Cousson, and W. Paulus, Chem. Phys. 226, 1 (1998).

${ }^{24}$ J. Meinnel, M. Mani, A. Cousson, F. Boudjada, W. Paulus, and M. R. Johnson, Chem. Phys. 261, 165 (2000).

${ }^{25}$ O. Kirstein and M. Prager, Appl. Phys. A: Mater. Sci. Process. 74, S1326 (2002) 